\title{
A Novel, Low-Cost Anesthesia and Injection System for Zebrafish Researchers
}

\author{
Yiğit Oskay, Barbaros Çetin,, Naz Şerifoğlu, ${ }^{2-4}$ Ayça Arslan-Ergül, ${ }^{2-5}$ and Michelle M. Adams ${ }^{2-4,6}$
}

\begin{abstract}
In this study, we designed and developed a novel low-cost system for anesthetizing and injecting adult zebrafish. The system utilizes a gradual cooling method for the anesthesia and maintains the fish in a stable anesthetic plane, as well as stabilizes the animal so that intraperitoneal injections can be consistently performed. It is a system that any laboratory with access to a workshop can build for their group. Moreover, it is a safe system for researchers, as well as a reliable one for repeated experiments since multiple fish can be injected quickly and there is little physical contact necessary between the investigator and the animal. This will likely reduce any unnecessary stress in the fish, as compared with manual methods of injection. Finally, the system is adaptable so that as the investigators' procedural needs change due to different research questions, that is, gradual rewarming or something of that nature, it could be modified.
\end{abstract}

Keywords: injection bed system, Peltier coolers, gradual cold water anesthesia, intraperitoneal injection

\section{Introduction}

$\mathbf{T}$ HE ZEBRAFISH IS AN IDEAL MODEL organism for a variety of biomedical research areas that focus on such things as neurodegenerative diseases and cancer. For many studies, a drug or a chemical is required to be introduced into the organism and the route of administration may be an intraperitoneal, intramuscular, or subcutaneous injection. Independent of the type of injection of interest, the animals must be anesthetized and stress levels kept to a minimum to provide them proper care.

For intraperitoneal injections into zebrafish, this can be done with the help of a net and a Hamilton syringe, ${ }^{1,2}$ either with anesthesia from tricaine methanesulfonate (tricaine, MS-222) $)^{1,2}$ or by gradual cooling. ${ }^{3}$ Our laboratory, as a routine practice, has adopted the method by Kinkel ${ }^{3}$ for intraperitoneal injections of bromodeoxyuridine ${ }^{4,5}$ because tricaine has adverse effects on the brain due to depressed respiration. ${ }^{6}$ However, the current netting procedure used in conjunction with the Hamilton syringe is likely causing more stress than is necessary for the animals, which is a very important consideration when doing injections into older animals. Moreover, cooling the fish by hand using ice water does not provide the opportunity for a consistent gradual cooling anesthetic effect. Thus, we set out to determine if there was a low-cost alternative that could be used.

Recently, a low-cost intraperitoneal injection set-up has been developed that emphasized the need for such systems for zebrafish researchers. ${ }^{7}$ However, this system does not include a process for utilizing anesthesia by gradual cooling and requires continuous animal contact. Moreover, performing a proper injection into a zebrafish requires three different subprocedures, which are consistency of the anesthetic plane of the animal, as well as proper stabilization of the syringe, and injection into the fish. Any of these procedures done in a usual aquarium set-up requires continuous physical contact between the investigator and the zebrafish.

In this article, we present detailed information about an aquarium that has been designed for an injection process with minimal contact between the fish and operator, as well as utilizing a gradual cooling method of anesthesia that provides a consistent anesthetic plane for every animal. To anesthetize the fish, instead of using a chemical such as tricaine, the water has been cooled down using thermoelectric coolers. With this system, the operator, with little to no animal handling, can

\footnotetext{
${ }^{1}$ Mechanical Engineering Department, İ.D. Bilkent University, Ankara, Turkey.

${ }^{2}$ Interdisciplinary Graduate Program in Neuroscience, Aysel Sabuncu Brain Research Center, Bilkent University, Ankara, Turkey.

${ }^{3}$ UNAM-National Nanotechnology Research Center and Institute of Materials Science and Nanotechnology, Bilkent University, Ankara, Turkey.

${ }_{5}^{4}$ Department of Molecular Biology and Genetics Department Zebrafish Facility, Bilkent University, Ankara, Turkey.

${ }^{5}$ Hacettepe University, Transgenic Animal Technology A.R.C, Ankara, Turkey.

${ }^{6}$ Department of Psychology, Bilkent University, Ankara, Turkey.
} 
make an injection into three stably anesthetized fish at the same time in less than $1 \mathrm{~min}$. This should minimize any major unnecessary stress for the animals. As compared with older methods, the biggest advantage is the simplicity and the reliability of the injection process, as well as the lost cost for the investigator to replicate such as system in their laboratory.

\section{Methods}

\section{Design of the integrated anesthesia and injection aquarium system}

For the design of the new system, it was necessary to consider that water has to be gradually cooled and maintained at a consistent temperature, which is below $12^{\circ} \mathrm{C}$ for anesthetizing the fish. Although refrigeration cycles based on the flow of a refrigerant in a closed vapor compression cycle is a conventional way to achieve cooling of a reservoir, they are not a feasible solution for the cooling of rather small spaces considering the efficiency and cost of these cycles, and the occupied space by the components of the cycle for the cooling of a small volume. On the other hand, despite the fact that thermoelectric coolers, which operate based on the Peltier effect and are known as Peltier coolers, have a considerably lower performance compared with the conventional refrigeration cycles, and considering the simplicity, reliability, and the cost, Peltier coolers become a feasible solution for the cooling of small volumes. ${ }^{8}$

A Peltier cooler is a simple solid-state device that generates a temperature difference, that is, a temperature gradient, between its opposite faces through the control of the electron velocity among the two types of semiconductors by the application of a potential difference. Furthermore, Peltier coolers have no moving parts, which would not induce mechanical vibration on the system, are maintenance free, and do not require any toxic refrigerant to operate. A basic demonstration of the thermoelectric module is given in Figure 1.

When a Peltier cooler is connected to a DC electric supply, one of its faces gets colder, whereas the other one gets hotter. However, the heat removed from a reservoir through the cold surface, needs to be rejected to another thermal reservoir through a hot surface. Typically, the hot surface is exposed to ambient air or a water bath. To achieve lower temperature values on the cold surface, a sufficient discharge of the heat from the hot surface is needed, which may require a forced convection, that is, an induced flow of ambient air or the fluid inside the water bath, rather than a natural convection. Moreover, a fin structure may be needed to extend the heat transfer area and enhance the heat transfer. Considering the volume of the water tank that has to be cooled for this aquarium system, Peltier coolers were utilized to cool down the water temperature. Furthermore, the vibration-free operation of the Peltier coolers helped ensure a pulsation-free operation of the whole system, hence avoiding any possible movement-induced disturbance of the fish.

This aquarium system that was designed consists of three main subsystems and these are the cooling, bed, and injection portions. Each of these systems was intended to complete the processes of anesthesia and injection with minimal physical contact between the fish and investigator. The whole system was designed to be made of aluminum to prevent any oxidation reaction due to the presence of water. During the design process the aim was to keep the pool volume as small as possible to decrease the energy and time that is required to cool the water to the desired temperature, which is below $12^{\circ} \mathrm{C}$. Although the pool volume is small, three fish can be anesthetized and injected at the same time.

There are only two moving parts in the aquarium, and these pieces are the bed and the shaft that carries the bed. Since these parts move after these fish are anesthetized, any physical harm due to a potential collision between the animal and a moving piece will not happen. A general view of the system is given in Figure 2A. As was mentioned previously, this aquarium system was designed to consist of three main subsystems and these are the cooling with an aquarium tank, bed, and injection portions (Fig. 2B). The designs of the subsystems are explained in the following sections.

Cooling subsystem. As explained in the first part of the Methods section, Peltier coolers have been utilized as a cooling unit. An efficient cooling is achieved as long as sufficient heat is removed through the hot face. To remove the heat from the hot face, water cooler units were designed. The cold surface of the Peltier is in contact with the outer surfaces of the aquarium, whereas the hot surfaces are in contact with the water coolers. To decrease the thermal contact resistance between the touching surfaces, a thermal paste is applied to both faces of the Peltier coolers. To enhance the effectiveness of the heat transfer in the water coolers, two baffle structures were integrated in the water coolers. Detailed side view and inner view of the Peltier coolers are given in Figure 3A and B, respectively.

Bed subsystem. The bed subsystem was designed so that once the fish becomes anesthetized, they would be stable

FIG. 1. Demonstration of the Peltier's working principle. An inner view of the Peltier with the cold (blue) and hot (red) surfaces on it. The P-type and the N-type semiconductors are illustrated and indicate the flow of electrons. The direction of the flow of the electrons in the semiconductors is demonstrated with the arrows. Color images available online at www

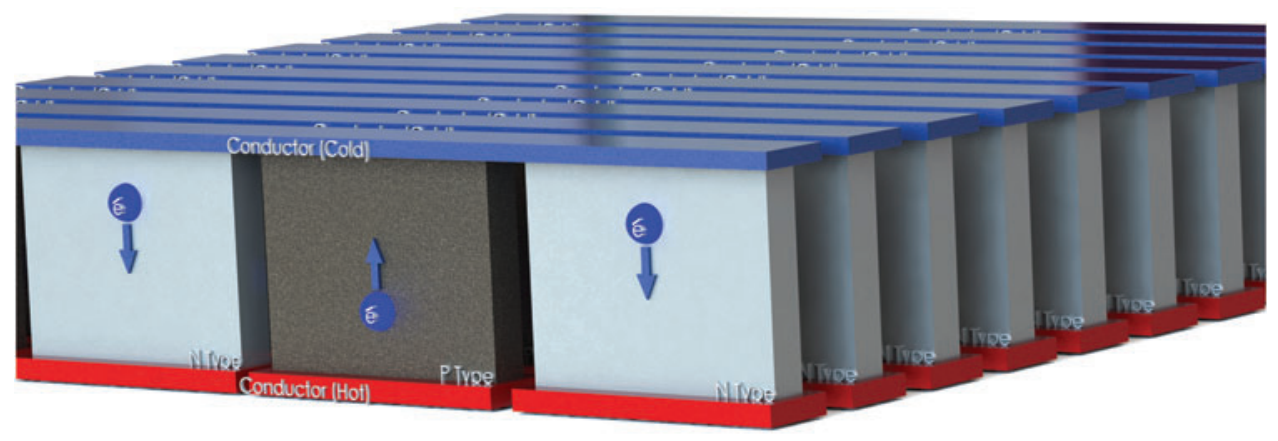
.liebertpub.com/zeb 

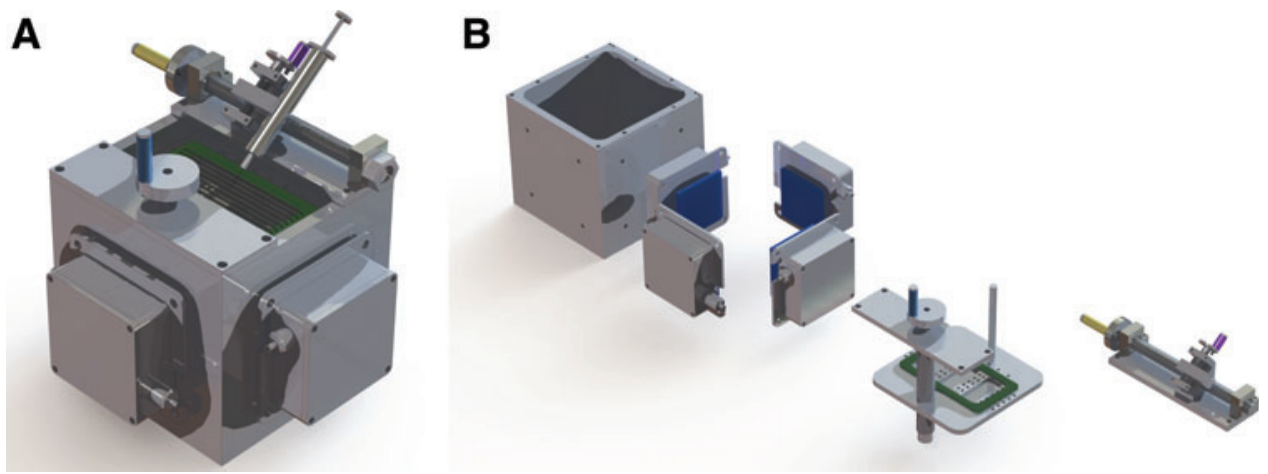

FIG. 2. Demonstration of the completed system and subsystems. (A) A picture of the entire injection system is shown. All of the subparts of the system are demonstrated in this figure, including the cooling subsystem with the main tank (aquarium), bed subsystem, and injection subsystem. Additionally, all controllers (rotator handles) are visible. (B) The subsystems are illustrated in this picture. From left to right: main tank (aquarium), bed subsystem, injection subsystem, and cooling subsystem. Color images available online at www.liebertpub.com/zeb

enough to receive an injection. An aluminum bed, which elevates in the aquarium, was designed to be used to keep the fish close to surface so that the needle may be aimed easily at their peritoneum (Fig. 4A). The design of the bed was such that it contains holes to permit the water to flow under it while the bed elevates (Fig. 4A). Any of the holes or gaps between the bed and the inner surfaces of the aquarium were intended to be tight enough so that the fish are not able to swim under the bed. Moreover, since the bed was devised to be elevated, the fish get closer to the surface in any case and would not be able to swim under the bed. The control of the bed elevation is
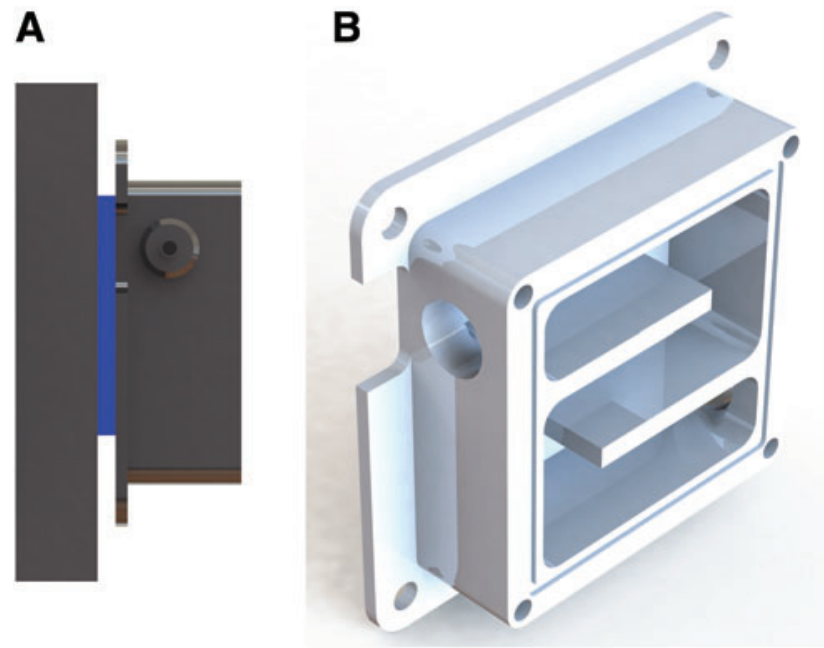

FIG. 3. Cooling subsystem (side view) and Peltier cooler (inner view). (A) A side view of the cooling subsystem is given. The blue rectangle indicates the Peltier portion of the cooler with its left side (cold side) in contact with the aquarium's outer wall and right side (hot side) in contact with Peltier cooler. (B) The inner view of the Peltier cooler is shown and the flow path of the cooling water is observed. The path is as follows: the water enters into the cooler from bottom right and leaves the cooler from the left top hole. The two inner walls inside the Peltier cooler that were added to increase the heat dissipation are illustrated in the photograph. Color images available online at www.liebertpub.com/zeb done by the rotation of the height controller (rotator with the violet handle), which is shown in Figure 4A and B.

Injection system. The injection system was designed to enable the needle move in two possible directions. The first one is the same direction with the needle that is pointing toward the body of the fish. By turning the injection controller (rotator with the pink handle; Fig. 4A, B) the needle would move toward the peritoneum of the fish and eventually penetrate the its body, and then be removed from the belly and move away from the animal. The other controller on the injection manipulator system was designed to be used to inject different fish held in parallel to each other on the bed. By turning the lateral movement controller (rotator with the yellow handle; Fig. 4A, B), the location of needle would be positioned so that the injection can be performed subsequently for three different fish. Since this injection system was planned to be used with this specific bed design, an injection system with two directions was enough. With these two controllers, it is possible for the investigator to do injections into three fish within less than $1 \mathrm{~min}$. A detailed view of the injection subsystem is given in Figure 4A and B.

\section{Construction of the integrated anesthesia and injection aquarium system}

A list of components is given in Table 1, and for the design of this system, with the exception of the blanket and the injector holder, all of these manufactured parts are required to be made of aluminum, preferably with series higher 4000 for easy machining. The manufacturing of the parts would need to be performed by conventional machining processes. For the machining of the injection subsystem, however, a computer numerical control (CNC) milling process is required since high precision machining is vital for the assembly step.

In the following paragraphs details are given about the injection system's manufacturing process and assembly, including any additional parts that were needed for its final construction. In Figure 5, every individual component of the system is shown and the list of these components in this figure used to assemble the system is given in Table 1. Since the machining process of the components of the system requires mostly $\mathrm{CNC}$ machining 

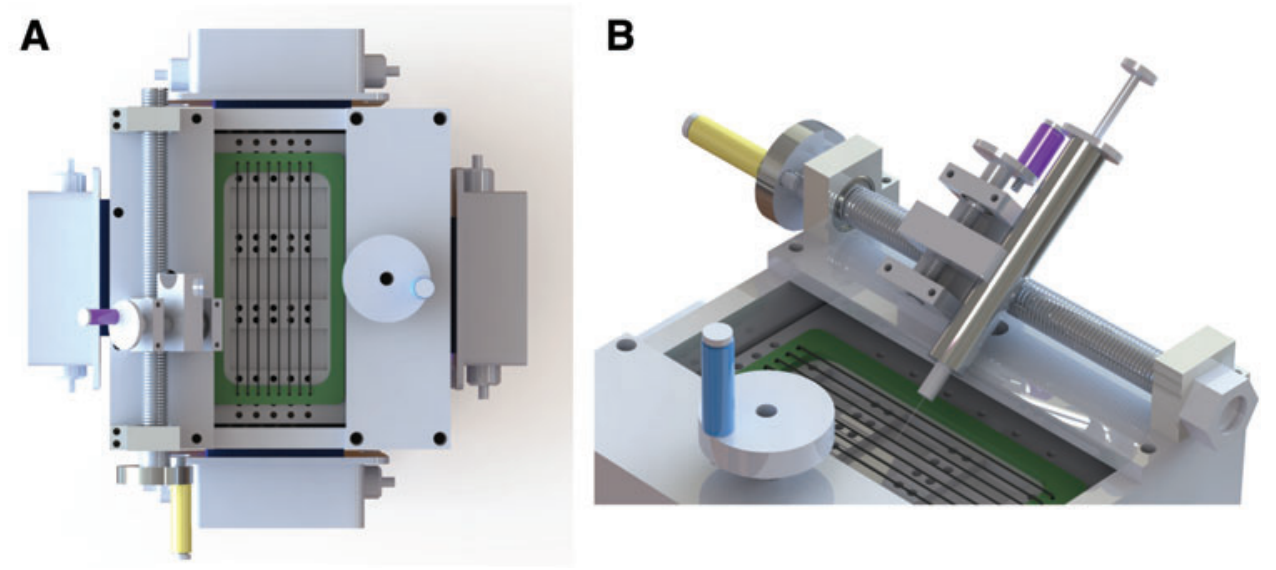

FIG. 4. Bed subsystem (top view) and the controllers of the injection and bed subsystems. (A) The bed subsystem is presented from the top viewpoint. The green rectangle indicates the blanket that keeps the fish steady during the injection and prevents any unexpected movement of the animal. The four Peltier coolers that are attached to the wall of the aquarium and the bed are also observed. The controller that elevates the bed with the fish is indicated by the violet-colored handle. (B) All controllers that are included in both the injection and bed subsystems are illustrated in this picture. The rotator with the yellow handle controls the syringe's lateral position, the one with the pink handle sets the location of the syringe toward or away from the belly of the animal, and the one with the purple handle sets the height of the bed. Color images available online at www.liebertpub.com/zeb

tools, manufacturing of these components is required to be performed in a professional machine shop.

Manufacturing process of the system. Every dimension and all required technical specifications of the manufactured components are given in this link: https://grabcad.com/ library/a-novel-low-cost-anesthesia-and-injection-systemfor-zebrafish-researchers-1. These technical drawings, which are found in the CAD files, will provide enough information for the manufacturing of the aquarium, such as locations and the diameters of the holes, distances, and thickness. Also, the general dimensions of the components are given

\section{Table 1. List of Components Given in Figure 5}

\begin{tabular}{|c|c|c|c|}
\hline Component number & Component name & $\begin{array}{l}\text { To which subsystem } \\
\text { the component belongs }\end{array}$ & Source material and dimensions \\
\hline 1 & Main tank & Main tank & Aluminum; $98 \times 98 \times 93 \mathrm{~mm}$ \\
\hline 2 & Bed & Bed subsystem & Aluminum; $84 \times 84 \times 4 \mathrm{~mm}$ \\
\hline 3 & Bed leadscrew & Bed subsystem & Aluminum; $r=12 \times 99.2 \mathrm{~mm}$ \\
\hline 4 & Blanket & Bed subsystem & $\begin{array}{l}\text { ABS or any } 3 \mathrm{D} \text { printing material; } \\
72.8 \times 38.8 \times 4.75 \mathrm{~mm}\end{array}$ \\
\hline 5 & Bed leadscrew holder & Bed subsystem & Aluminum; $98 \times 30 \times 4 \mathrm{~mm}$ \\
\hline 6 & Bed leadscrew head & Bed subsystem & Aluminum; $r=25 \times 6 \mathrm{~mm}$ \\
\hline 7 & Bed and lateral leadscrew shell & Bed subsystem & Aluminum; $r=6 \times 19.5 \mathrm{~mm}$ \\
\hline 8 & Bed and lateral screw arm & Bed subsystem & Aluminum; $r=5.5 \times 29.5 \mathrm{~mm}$ \\
\hline 9 & Bed enhancer & Bed subsystem & Aluminum; $r=5 \times 92.5 \mathrm{~mm}$ \\
\hline 10 & Peltier Cooler & Cooling subsystem & Aluminum; $65 \times 65 \times 18 \mathrm{~mm}$ \\
\hline 11 & Peltier cooler Cap & Cooling subsystem & Aluminum; $51 \times 51 \times 3 \mathrm{~mm}$ \\
\hline 12 & Couplings & Cooling subsystem & From market \\
\hline 13 & Peltier & Cooling subsystem & Peltier; $40 \times 40 \times 2 \mathrm{~mm}$ \\
\hline 14 & Lateral rail & Injection subsystem & Aluminum; $98 \times 30 \times 4 \mathrm{~mm}$ \\
\hline 15 & Lateral leadscrew & Injection subsystem & Aluminum; $r=22 \times 113 \mathrm{~mm}$ \\
\hline 16 & Bed and lateral leadscrew shell & Injection subsystem & Aluminum; $r=6 \times 19.5 \mathrm{~mm}$ \\
\hline 17 & Bed and lateral screw arm & Injection subsystem & Aluminum; $r=5.5 \times 29.5 \mathrm{~mm}$ \\
\hline 18 & In-out rail & Injection subsystem & Aluminum; $20 \times 20 \times 15 \mathrm{~mm}$ \\
\hline 19 & Syringe holder & Injection subsystem & $\begin{array}{l}\text { ABS or any 3D printing material; } \\
22 \times 12 \times 7 \mathrm{~mm}\end{array}$ \\
\hline 20 & In-out bearing & Injection subsystem & Aluminum; $r=9 \times 2.5 \mathrm{~mm}$ \\
\hline 21 & In-out bearing holder & Injection subsystem & Aluminum; $15 \times 10 \times 4 \mathrm{~mm}$ \\
\hline 22 & In-out leadscrew & Injection subsystem & Aluminum; $r=14 \times 32.5 \mathrm{~mm}$ \\
\hline 23 & In-out leadscrew shell & Injection subsystem & Aluminum; $r=4.5 \times 9.8 \mathrm{~mm}$ \\
\hline 24 & In-out leadscrew arm & Injection subsystem & Aluminum; $r=5 \times 25 \mathrm{~mm}$ \\
\hline 25 & Lateral bearing holder & Injection subsystem & Aluminum; $20 \times 16 \times 7 \mathrm{~mm}$ \\
\hline 26 & Lateral bearing & Injection subsystem & Aluminum; $r=12 \times 7 \mathrm{~mm}$ \\
\hline
\end{tabular}




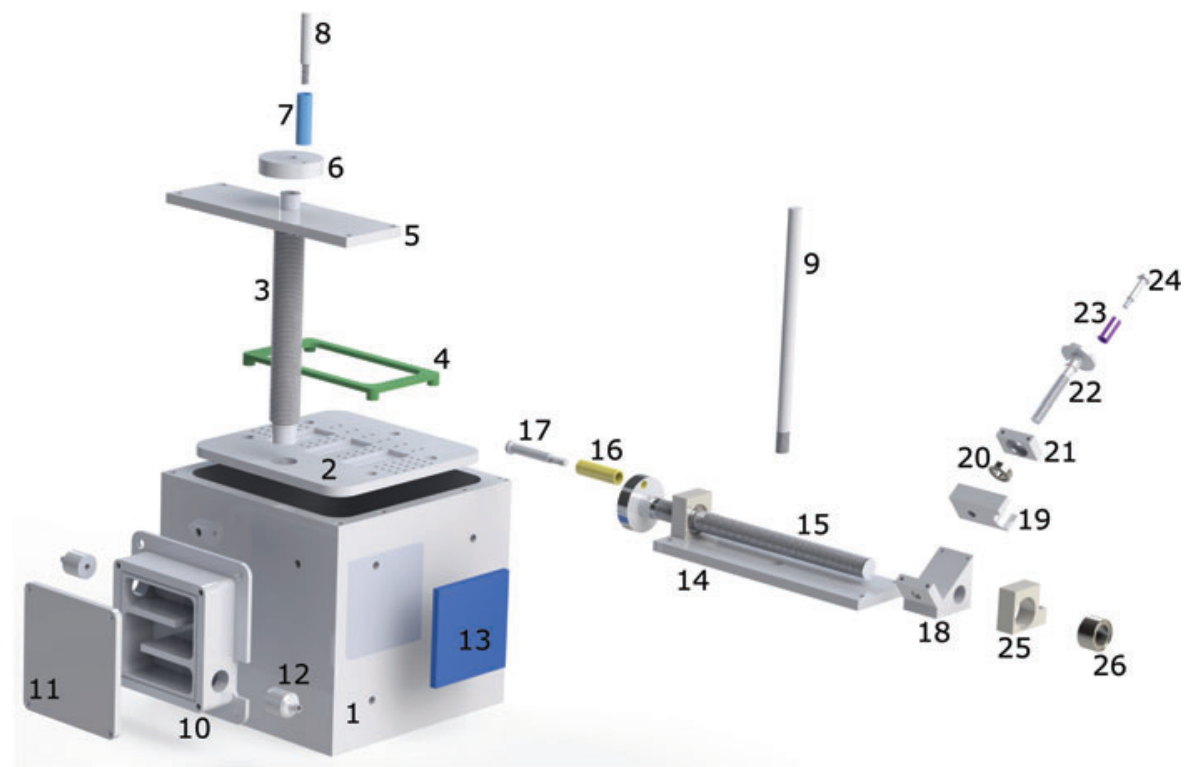

FIG. 5. Individual components of the entire anesthesia and injection system. One of each of the individual components is illustrated in this figure and it is indicated how the general assembly is made. They are numbered and in Table 1 information is presented that indicates each component name, material, and to which subsystem they belong. Please refer to Table 1 for this information. Color images available online at www.liebertpub.com/zeb

in Table 1 to permit the reader roughly to imagine the size of them.

(1) In the first step, the main tank (Component 1; Fig. 5) is manufactured. An aluminum cube with $110 \mathrm{~mm}$ side was machined using a CNC milling machine. First, the main aquarium hole and bed system's hole was required to be machined, and then the holes at the top of the aquarium were drilled. During the very last step of machining process, the holes at the side surfaces of the aquarium, where the Peltier coolers would be attached, needed to be drilled.

(2) During the second step, Peltier coolers (Component 6; Fig. 5) are manufactured with a $\mathrm{CNC}$ milling machine. For a Peltier cooler, a raw aluminum block with dimensions of $75 \times 75 \times 24 \mathrm{~mm}$ is required. Alternatively, if an aluminum block with dimensions of $320 \times 320 \times 24 \mathrm{~mm}$ is available, and if the machining capacity of the milling is higher than $320 \times 320 \mathrm{~mm}$, it is possible to manufacture four Peltier coolers from a single piece of raw material.

The manufacturing process needed to begin with the machining of the top surface of the Peltier coolers, which included the baffles, the O-ring channel, and the holes for the screws. Next, the coupling could be drilled by machining the sides of the Peltier coolers. If the four Peltier coolers could be manufactured in a single step, this would provide a considerably time-saving point in the manufacturing process. Following this step, the Peltier cooler caps (Component 7; Fig. 5) were manufactured from an aluminum plate with dimensions $220 \times 220 \times 5 \mathrm{~mm}$ using A CNC milling machine.

(3) For the third step, the injection system is manufactured. First, the lateral rail (Component 10; Fig. 5) is made by cutting a piece of raw material with dimensions $8 \times 105 \times 35 \mathrm{~mm}$ using the CNC milling machine (DECKEL MAHO-HSC55). Apart from the injector holder rail, it is possible for the parts of the injection system that do not have a cylindrical geometry to be produced in a single step from one sin- gle piece of raw material. Therefore, one in-out rail (Component 12; Fig. 5), two lateral bearing holders (Component 14; Fig. 5), and two in-out bearing holders (Component 17; Fig. 5) can be manufactured from one piece of raw material with dimensions greater than $150 \times 150 \times 20 \mathrm{~mm}$. All of these components require milling from both the top and side faces of each of these work pieces.

(4) During the fourth step, the bed screw leader (Component 3; Fig. 5), lateral screw leader (Component 18; Fig. 5), in-out screw leader (Component 15; Fig. 5), bed enhancer (Component 5; Fig. 5), and the controllers (Components 19-21; Fig. 5) are made using a lathe machine. Both the bed screw leader and lateral screw leader are machined from a single cylindrical work piece with a diameter of $30 \mathrm{~mm}$ and length of $250 \mathrm{~mm}$. The in-out screw leader, bed enhancer, and the controllers are produced from a work piece that has a diameter of $12 \mathrm{~mm}$ and length of $180 \mathrm{~mm}$ using the lathe machine.

(5) For the final step, the remaining components, which are injector holder (Component 11; Fig. 5) and blanket (Component 4; Fig. 5), are printed with a 3D printer since high-level precision is not required. However, a high infill percentage needs to be used for the 3D printing to obtain an appropriate product. After the $3 \mathrm{D}$ printing of the blanket, by using a sewing yarn, the net can be knitted between the holes by using a sewing needle to produce the net which is made of sewing yarn. Peltiers (Component 8; Fig. 5), couplings (Component 9; Fig. 5), bolts, nuts and bearings are purchased from the local market.

It may be necessary to make modifications to the dimensions and the tolerances, which are the acceptable manufacturing errors, of such components as couplings, bolts, o-rings and bearings. This is due to consideration of the manufacturing capabilities of the reader's local machine shop and the market standards of the components that will be bought from the reader's local stores. 
Manufacturing services. In situations where there is a lack of machine shop services in the reader's research facility, he or she may use the services of the following companies listed with their corresponding websites:

North America: Baron Machine (www.baronmachine.com) South America: Pillar Machine (www.pillarmachine.com)

Asia: Source in Asia (www.sourceinasia.global)

Europe: Proto Labs (www.protolabs.co.uk/about-us/ locations)

Africa: Vomak Industries (www.vomak.co.za/index.html)

Australia: D\&N Engineering Supplies (http://eng.com.au)

\section{Assembly process of the system}

(1) The first step of the assembly is connecting the bed subsystem to the main tank (Component 1; Fig. 5) because following the fixing of the injection subsystem to the main tank, it would be impossible to reach in the aquarium to install the bed subsystem. The bed enhancer (Component 5; Fig. 5) and bed screw leader (Component 3; Fig. 5) need to be connected to their locations, then the bed (Component 2; Fig. 5) can be attached to them.

(2) Once the bed subsystem is installed, the injection subsystem can be constructed. This process begins with attaching the lateral rail (Component 10; Fig. 5) to the main tank. However, before attaching anything to the main tank, the in-out mechanism of the injection subsystem has to be assembled.

(3) The in-out bearings (Component 16; Fig. 5) must be attached to the in-out bearing holders (Component 17; Fig. 5), and then these holders should be fixed to the in-out rail (Component 12; Fig. 5) with 2 bolts for each holder. At this point it is possible to attach the injector holder (Component 11; Fig. 5) to the in-out screw leader (Component 15; Fig. 5) through the hole on the injector holder.

(4) At this point, the components numbered 11, 12, 15, 16, and 17 are connected to each other. Following this step, the lateral bearing holders (Component 14; Fig. 5) must be attached to the lateral rail (Component 10; Fig. 5), and then the lateral bearings (Component
13; Fig. 5) are fixed to the holders. The same assembling steps utilized for the components numbered 11 , 12,15 , and 17, must be applied in the same order to the components numbered 12,10,18, 13, and 14 .

(5) Now that the injection subsystem is fully assembled, the cooling subsystem must be put together. Each Peltier cooler (Component 6; Fig. 5) has to be connected to their caps (Component 7; Fig. 5) before they are attached to the main tank. Between the cap and the Peltier cooler an O-ring must be installed to the O-ring groove on the Peltier cooler to avoid any leakage. In each country's local markets there will be O-rings of different standards such as ISO or ANSI. Therefore, it would not be useful to specify a certain O-ring because it is possible that the reader may not find the given O-ring in his/her local market. It is better to use the advised O-ring by the dealer of the local market, or the machine shop. After installing the O-rings, then the caps can be attached to the Peltier coolers.

(6) Before inserting the Peltier coolers (Component 8; Fig. 5) to the main tank, both the hot and cold faces must be covered with a thermal paste and the electric cables of the Peltiers must go down along the side walls of the main tank. The hot side of the Peltier must be in contact with the Peltier cooler and the cold side of the Peltier must be in contact with the main tank. At this point, the Peltier coolers, with 4 bolts per cooler, can be attached to the main tank.

(7) For the final step, all couplings (Component 9; Fig. 5) must be attached to the Peltier coolers and then elastic pipes can be fixed to these couplings. The Peltier coolers must be connected to each other in a serial manner with elastic water or pneumatic pipes. After connecting two Peltiers to each other in parallel and joining these two Peltiers to the power supplies, the system is done. It is important that each power source is connected to two Peltiers in parallel and it is important that the cables be covered very well with insulators to avoid any electric short out. The final assembled system and magnified view of the injection portion is given in Figure $6 \mathrm{~A}$ and $\mathrm{B}$, respectively.
A

FIG. 6. Assembled aquarium. (A) In this image, the final version of the completed system is seen. (B) In the second image a close-up version of the injection bed is observed. The precision of the finalized system can be seen. Color images available online at www.liebertpub.com/zeb

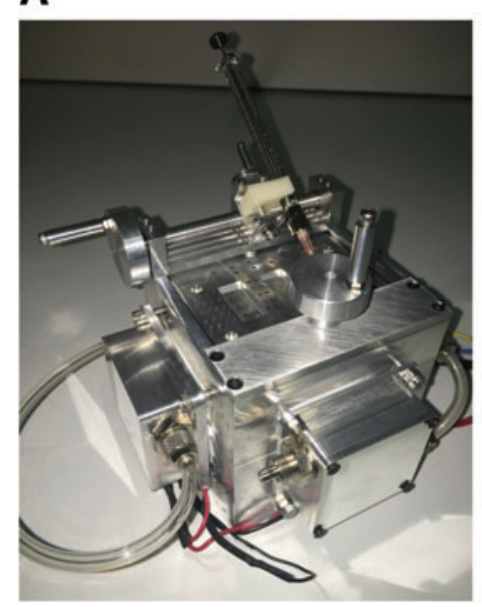

B

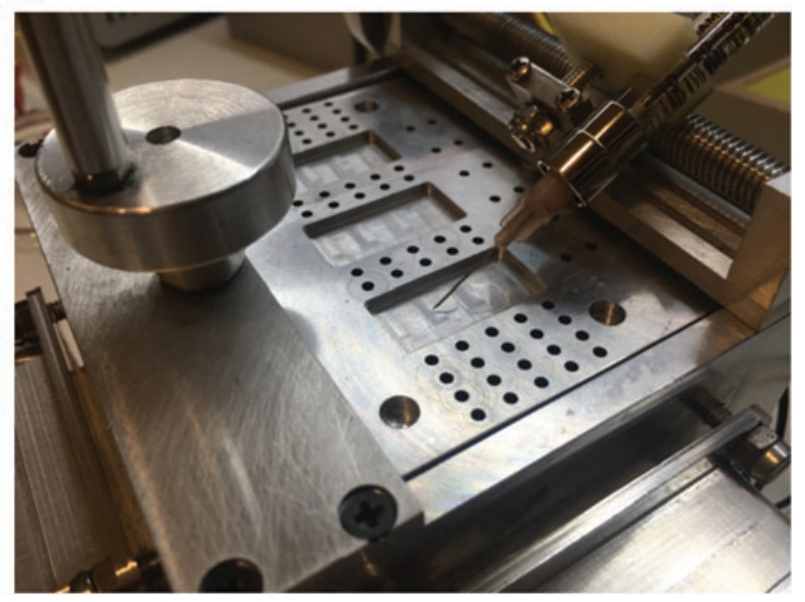


The following paragraphs give more specific information about the materials used in the description of the final system assembly that was described previously.

Thermal paste application. Each face of the Peltier coolers has to be covered with thermal paste to increase the effectiveness of the heat transfer. A dramatic reduction in the time it takes for the cooling to occur will be achieved following the application of the thermal paste.

Sealing element. Since Peltier coolers are manufactured in two pieces, most likely a leakage will occur if there is no sealing element between these pieces. To avoid leakage, an O-ring needs to be placed between the matching surfaces of the Peltier coolers.

Tubing. Peltier coolers are connected to each other serially. After the water comes from the sink or from any other water source, it flows through the water coolers. Using flexible tubing is important since it lets the experimenter operate the system in any area of the laboratory. Moreover, if two valves are also installed on the system, one to the inlet of the first cooler and the other one to the outlet of the last cooler, it is possible to rotate, wash, and store the system without spilling the water.

Injector holder. This part of the system is manufactured for a specific syringe size by a 3D printer. By considering different diameters of different syringes, different injector holders can be designed. One has to pay attention to the size of the holder radius in the designing of an injector holder since the friction between the syringe and the holder must be high enough to hold the syringe firmly, but its removal also has to be facilitated.

Power supply. Four Peltiers, each with a power of $80 \mathrm{~W}$, are used and connected parallel to each other. To provide the required electric power, the system needs two DC power supplies with a power amount of $200 \mathrm{~W}$ and each power supply is used to provide the two Peltier coolers with the required capacity that is necessary. This means that each power supply, which can provide $200 \mathrm{~W}$, has been used for two Peltiers, which requires $160 \mathrm{~W}$ in total. The overall power used to run the system is $320 \mathrm{~W}$, whereas the total power that can be generated by the supplies is $400 \mathrm{~W}$.

If the reader cannot find the exact same Peltier with the 80 $\mathrm{W}$ power, the reader can use another four Peltiers, each of which has a power of at least $80 \mathrm{~W}$. The type of the Peltier used in the current apparatus is "TEC-12708." For this specific Peltier, it is advised to use a $12 \mathrm{~V}$ energy supply so that the maximum potential difference that can be generated by the supplies is $12 \mathrm{~V}$. In the case of utilizing different Peltiers, it is important not to use any power supply that generates more potential difference than the Peltier's maximum working potential difference.

Blanket. A blanket is needed to keep the fish stable during the injection. In the given link (https://grabcad.com/library/ a-novel-low-cost-anesthesia-and-injection-system-for-zebrafishresearchers-1), the 3D printing files can be found for use in 3D printing software to print the blanket. After the plastic part is manufactured, the net has to be knitted by hand in a manner so that it is not so tight, but tight enough to keep the fish under the blanket. The material that is used to make the net is sewing thread and by using the holes at the side of the blanket a net can be knitted between the holes.

\section{Procedures for use of the integrated anesthesia and injection aquarium system}

In this section, the experimental process of anesthetizing and injecting the animals is explained in a stepwise fashion using the test set-up illustrated in Figure 7. A Daqbook, which is an Ethernet-based, $16 \mathrm{Bit}, 200 \mathrm{kHz}$ data acquisition system, and a computer, as well as thermocouples were used in the experiments to obtain data presented in Figure 8. For ordinary experiments, it is not necessary to employ this equipment, but rather a very simple electronic thermometer could be used.

(1) Initially the aquarium has to be rinsed with tap water and cleaned to remove any potential dust that might be a contaminant for the animal. Following the rinsing with tap water, all of the surfaces of the system, that is, bottom and walls, which would come in contact with the fish during anesthesia and injection are cleaned with 70\% ethanol and allowed to air dry. During the rinsing period, it is important that the operator avoid soaking the Peltier or the electric sources in the water as this will render the system unusable. For this reason, first the machine must be unplugged to keep it from cooling down, and there is a safe side with no electrical cables, therefore, you pour the water from that side. If the water spills, the researcher can dry it with a tissue or towel.

(2) After aquarium is thoroughly cleaned, then it can be filled with appropriate water for the fish. For example, in the present study system water obtained from our Tecniplast housing system was utilized,

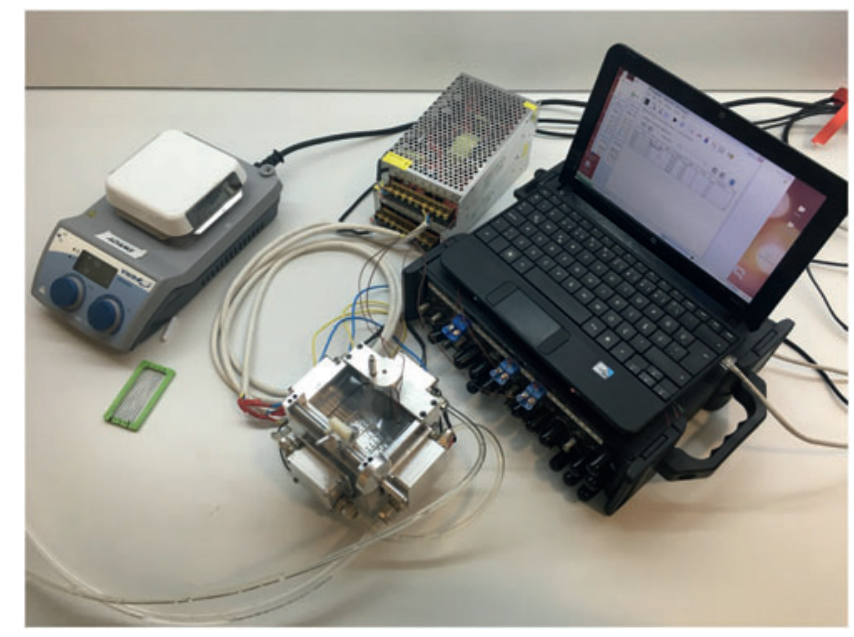

FIG. 7. Test set-up. The final test set-up is illustrated in this figure. In this figure, the Daqbook, which is an Ethernetbased, 16 Bit, $200 \mathrm{kHz}$ data acquisition system, and the computer, in addition to the anesthesia and injection system are observed. The Daqbook and computer are the equipment that was used to monitor continuous temperature data over time. The equipment on the top left of the figure is a stir plate that was used to see the effect of mixing the water. Color images available online at www.liebertpub.com/zeb 


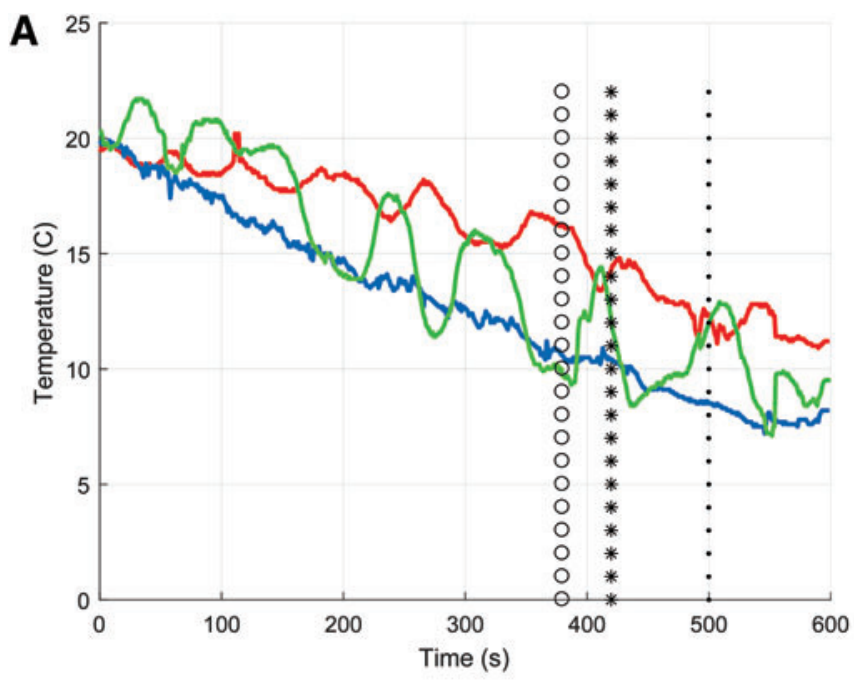

Experiment 1

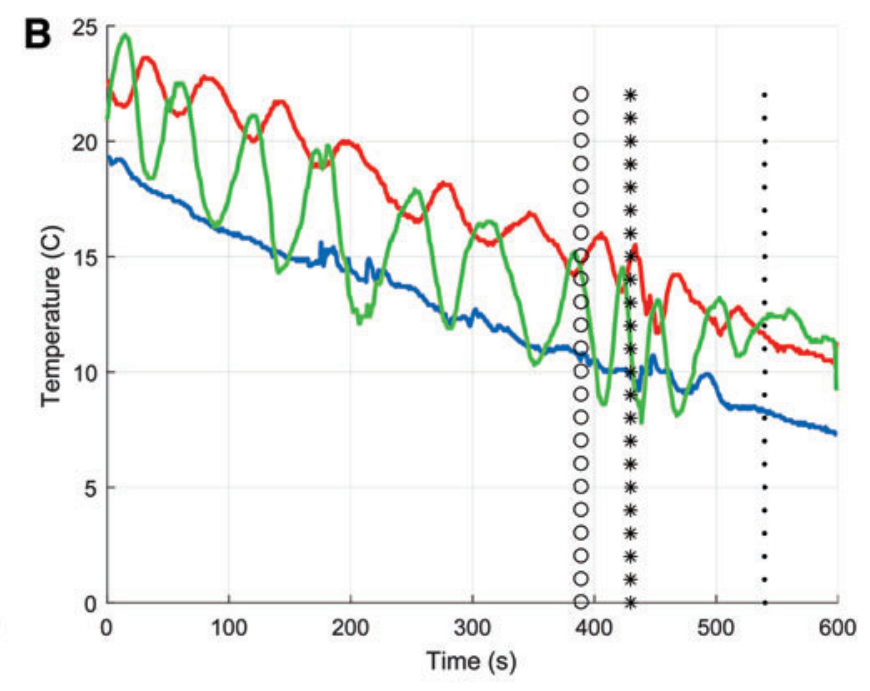

Experiment 2

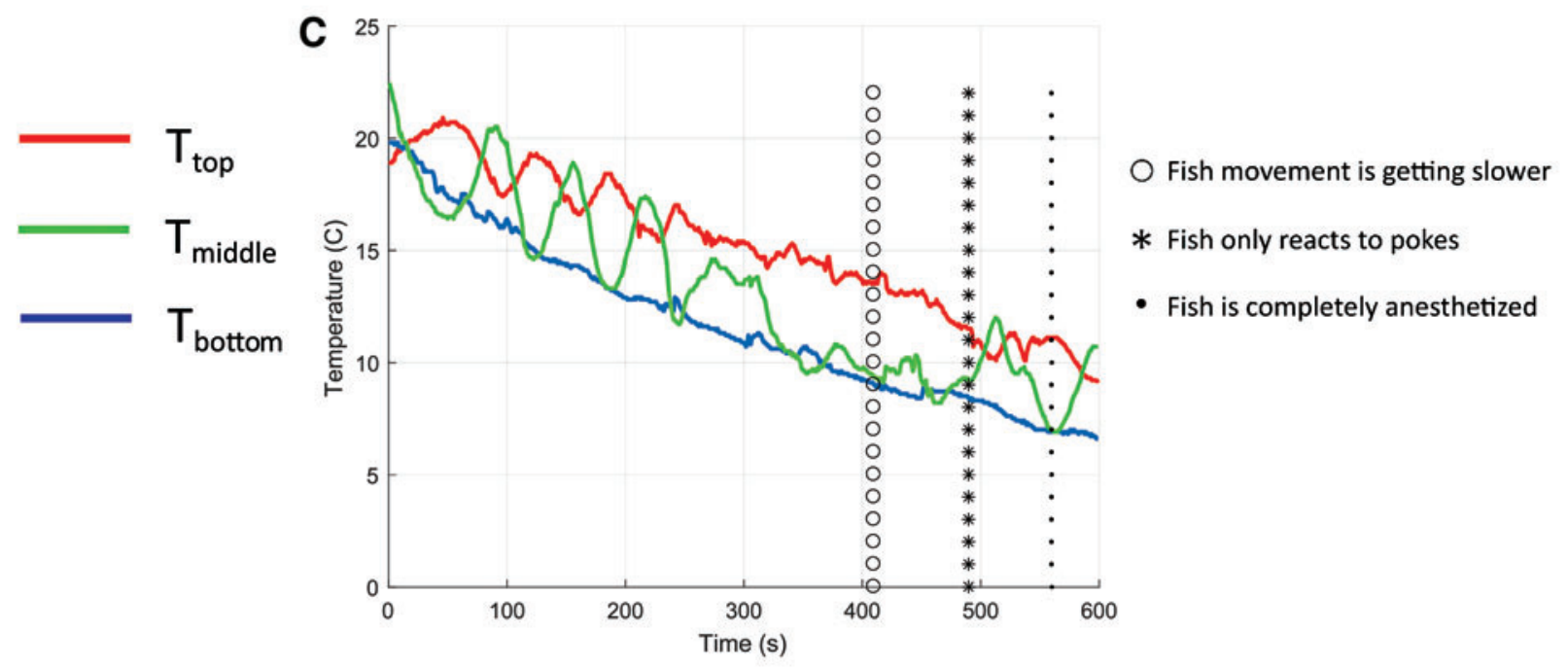

Experiment 3

FIG. 8. Results of the gradual changes in temperature across time. The three graphs labeled A, B, and C, which are illustrated in this figure, are representative of the behavior of three different fish across three separate trials. As was expected, the temperature cooled gradually over time and at $\sim 10^{\circ} \mathrm{C}-12^{\circ} \mathrm{C}$ the fish is anesthetized. All three trials had almost identical changes in the behavior of each animal over time so the system delivers a reliable and consistent form of anesthesia for the injection. The different colored lines provide temperature measurements across time at the surface of the water (red), middle of the water (green), and bottom of the water (blue). Color images available online at www.liebertpub.com/zeb

but it is possible that any suitable aquarium water be used. The water height should be no more than 4 or $5 \mathrm{~mm}$ below the top of the aquarium to avoid spilling.

(3) During this step, the incoming water pipe has to be connected to the water source, preferably to tap since the purity of the circulating cooling water is not important because there is no contact of that water with the fish. After the connection is made between the incoming water pipe and the tap, the outlet pipe must be directed for drainage.

(4) Once all of these connections are completed, the water circulation should be started, and then, after the water starts to circulate, the electric source must be activated. When the power source is plugged in and turned on, the cooling process begins.

(5) During the cooling process, the temperature of the water in the aquarium has to be measured to determine whether there is gradual cooling and the fish's behavior should be observed during this time. When the fish's movements become slower, this indicates that the animal is beginning to become anesthetized. Also, during the cooling process, if the water is thoroughly mixed in the aquarium, then a more uniform temperature will be created. To achieve this process, the water can be mixed with a Plastic Pasteur pipette. A magnetic stirrer cannot be used as it will stress and/or harm the fish. 
(6) Before making the injection, the syringe must be attached to the injector holder. The syringe may be attached following the start of the cooling process.

(7) Before anesthetizing the fish, they are kept in a regular fish tank or bowl (Supplementary Video; Supplementary Data are available online at www .liebertpub.com/zeb). To start the anesthesia and injection process, the fish are moved into the system with a regular fish net. Following this process, the system is turned on and as the temperature of the water decreases, the fish will begin to become immobile and anesthetized (Fig. 8 and Supplementary Video).

When the fish is fully anesthetized, the animal is brought closer to the surface for the injection process. To bring the fish closer to the surface, the elevation controller disk (rotator with the violate handle; Fig. 4A, B) should be rotated in a clockwise fashion so the bed will elevate. When bed reaches the required height, the net of the blanket (Component 4; Fig. 5) must be attached to fix the fish to its bed. This net prevents the fish from sliding around the aquarium when the needle is inserted into its peritoneum.

(8) When the fish's position is appropriate for the injection, the lateral movement disk controller (rotator with the yellow handle; Fig. 4A, B) can be rotated so that the needle's direction can be aligned with the fish. Then, the needle can be moved forward to be inserted into the fish by rotating the injection disk controller (rotator with the pink handle; Fig. 4A, B).

(9) After needle penetrates the fish, the drug can be injected to the fish's body by pushing the plunger of the syringe.

(10) At this point, the injection process is complete and to avoid any thermal shock to the fish, instead of taking it directly to a warm place, it is better to keep it in the cold aquarium and adjust the temperature by adding warm water into the aquarium while removing the cold water from it. It takes $\sim 5-10 \mathrm{~min}$ to warm up the system to help the animals recover from the anesthesia (Supplementary Video). There are two ways to help the fish recover. In the first procedure, the cold water is aspirated or vacuumed out of the system and warm water is replaced in the tank (Supplementary Video). As this process happens, the fish will recover quickly and then it should be moved back to its home tank. The second procedure requires the anesthetized fish to be removed with a net and put but into a recovery tank, which has warm water.

\section{Testing of the integrated anesthesia and injection aquarium system}

To test the applicability of the newly designed system, 23 zebrafish were anesthetized and injected using this machine. These data were compared with fish used with a previous protocol of intraperitoneal injection utilizing a sponge and data were reported in a previous study. ${ }^{5}$ All fish used currently and in the previous study ${ }^{5}$ were raised and maintained in the Bilkent University Zebrafish Facility. Fish were housed at $28^{\circ} \mathrm{C}$ on a 14 -h light:10-h dark cycle. All fish were of the
$\mathrm{AB}$ strain, and male or female animals within the age range of 3-24 months were used. Ethical permission for this study was obtained from the Bilkent University Local Animal Ethics Committee (HADYEK) with approval date: Feb 9, 2010 and no: 2010/1.

\section{Results}

The aim of our study was to design a low-cost novel anesthesia and injection system for zebrafish researchers. As was mentioned in the Methods section, we utilized Peltier coolers that would gradually cool the injection system water and help keep the anesthesia temperature consistent to maintain an adequate anesthetic plane during the injection process. This system would permit injections into three fish efficiently in one round and with minimal contact between the investigator and the animal, therefore, are very likely inducing minimal stress. Additionally, this method of using cold water as an anesthetic is the most widely accepted method now ${ }^{3,9}$ as tricaine methanesulfonate (tricaine, MS-222) has been shown to have adverse effects on the fish. ${ }^{9}$ Therefore, this system should be better for the animals, especially in the case of older fish, which are used very frequently by our group. Finally, this system is low cost for investigators to build in their own university or research institute.

Once the system was constructed, we tested whether the system would anesthetize our fish appropriately for peritoneal injections and that these injections would be able to be performed reliably. Our results demonstrated that for all trials, the temperature gradually decreased across time and an appropriate anesthetic plane was induced. The results of our tests are shown in Figure 8A-C and portions of them can also been observed in the supplementary video located in the following link, https:// drive.google.com/file/d/OBzzXuI_CZK3pRFc2WVg3YldYYWs/ view? usp=drive_web. We observed that across three different trials, using three different fish one in each trial, the temperature of the water started around $20^{\circ} \mathrm{C}-25^{\circ} \mathrm{C}$ and reduced gradually to $10^{\circ} \mathrm{C}-12^{\circ} \mathrm{C}$. At the temperature of $\sim 12^{\circ} \mathrm{C}$, the fish stopped moving and by $10^{\circ} \mathrm{C}$ or less the animal was completely anesthetized since it did not respond to a poke of a needle and the injection could be performed (Fig. 8A-C). After the injection of each of the fish, the water was then gradually warmed by adding fresh system water and removing the cooler water. After each trial when the fish recovered, they were returned to their home tank.

An important experiment to perform is whether or not the injection system may cause unwanted effects, such as not inducing a reliable and consistent anesthetic plane and the fish would not be able to be injected. This can be a problem when the injections are performed by hand using a sponge to anesthetize the animal. Therefore, we examined how the behavior of the fish changed during the gradual cooling process. As shown in Table 2, we observed that all the fish, whether they received an injection or not, moved into a consistent anesthetic plane, that is, no response to tactile stimuli, in a similar amount of time. This is in contrast to the behavior that is observed using the previous protocol, which can take between 1.5 and $20 \mathrm{~min}$ for each fish to reach that anesthetic plane. Moreover, as can be seen in the three representative trials in Figure 8A-C, the gradual temperature changes and corresponding behavioral changes were consistent with those expected using a gradual cooling method. Thus, our system is reliable and stable in inducing an 
TABle 2. Recovery Data of the Fish

\begin{tabular}{lccc}
\hline & No injection & $\begin{array}{c}\text { Intraperitoneal } \\
\text { injection }\end{array}$ & $\begin{array}{c}\text { Previous protocol of } \\
\text { intraperitoneal injection using sponge }\end{array}$ \\
\hline $\begin{array}{c}\text { Number of fish tested } \\
\begin{array}{c}\text { Average time required for } \\
\text { fish to be anesthetized }\end{array}\end{array}$ & 8 & 15 & 100 \\
$\begin{array}{c}\text { Response to tactile stimuli } \\
\text { after fish is anesthetized }\end{array}$ & $0 \%$ & $10 \mathrm{~min}$ & Between 1.5 and $20 \mathrm{~min}$ \\
\hline
\end{tabular}

anesthetic plane for intraperitoneal injections into zebrafish and the injections can be done very quickly into three fish, that is, in less than $1 \mathrm{~min}$. Therefore, our system likely induces very little stress into the animals that we inject.

\section{Discussion}

The goal of this work was to design an efficient and novel low-cost anesthesia and injection system that would cause minimal stress to the zebrafish. We used Peltier coolers because they are a solid-state device that would gradually cool the water without the use of any refrigerant chemicals that might pose a potential health risk to the animals. Moreover, we needed a device that would permit us to inject multiple stabilized fish quickly with as minimal contact as possible to minimize stress in the animals. We constructed an anesthesia and injection system from aluminum materials in our machine shop that contained three subsystems, including a bed, cooling, and an injection subsystem.

The results from our tests indicated that our system gradually cooled and maintained the water at an appropriate temperature based on NIH guidelines so that the fish became immobile and anesthetized. Following that state, the injection was made very easily and efficiently, allowing the fish to recover without inducing any unnecessary stress in the animal. With the current design, one round of anesthetizing three fish, injecting them, and having them recover takes $\sim 15-20 \mathrm{~min}$. Thus, this system is safe and most likely more beneficial for the animals than using hand injections and/or with standard chemical anesthetics.

Chemical anesthetics are used on zebrafish, but are not as good as the gradual cooling method with ice water. For example, tricaine methanesulfonate (tricaine, MS-222) is a widely used anesthetic for zebrafish, but in adult zebrafish it reduces heart rate and causes high mortality under long-term sedation. ${ }^{10}$ Usage of tricaine has come into discussion after a study found it to be an aversive compound, even at low concentrations. ${ }^{11}$ Similarly, another study found tricaine to be more harmful than metomidate hydrochloride and clove oil. $^{12}$ Isoflurane coupled with tricaine has been shown to lessen the effects of tricaine on the heart rate. ${ }^{10}$

Gradual cooling is a rapid and safe method of anesthesia that has been shown to be superior to tricaine as a method of euthanasia. It enables anesthesia in $10 \mathrm{~s}$, persist for up to $10 \mathrm{~min}$, and with zero mortality. ${ }^{13}$ In direct comparisons of chilled water with tricaine, it was shown that the duration effect of tricaine euthanasia took longer than chilled water and that fish regained consciousness while exposed to tricaine as opposed to chilled water. ${ }^{9}$ Survivability assays have shown that gradual cooling can be applied to larvae repeatedly. ${ }^{13}$

One shortcoming of gradual cooling is that all the surfaces and instrumentation that come in contact with the fish must be kept at low temperatures, ${ }^{14}$ which includes the hands of the researcher. So there is a need to design a system that cools water to an anesthetic plane, and the system that we have designed will do this and provide a more adequate anesthesia for the fish than chemical anesthetics.

In addition to the more harmful effects of chemical anesthetics, there is also the issue of stress that might be induced in the animals when being handled and prepared for injections. In the present system, there is minimal contact between the fish and the investigator. There is only the placement of the fish into the injection aquarium system and removing the fish following the anesthesia. Thus, any stress induced by netting the fish would be negligible and this is a very important factor as we are studying the brains of older fish, that is, animals greater than 18 months of age.

Therefore, we want to minimize any unnecessary stress from being induced that could cause an increase in cortisol levels leading to changes in the brain chemistry of these animals. Cortisol levels in zebrafish have been found to increase in response to stressors such as osmotic shock, ${ }^{15}$ dominant-subordinate interaction, ${ }^{16}$ and predator stress. ${ }^{17}$ In a previous study, our group demonstrated high cortisol levels following intraperitoneal injections in fish using a method of netting and then hand injections of anesthetized fish immobilized in a sponge. ${ }^{5}$ Interestingly, although there were no significant differences in cortisol levels in young versus old fish, numerically it seemed that older fish were more resistant to this injection stress than young, but doing the injections using this previous protocol with a sponge were not as consistent as those that are using system, such as we have designed. Thus, we are in the process of systematically determining whether there are lower cortisol levels in the fish that are used in the current system.

The current system is used to gradually cool the water to an appropriate anesthetic plane and three fish can be quickly injected with minimal interaction with the investigator. Following this process, the fish then recover with the rewarming of the water by adding room temperature and removing the cold water. With the current system, one round of anesthetizing three fish, injecting them, and having recovery takes $\sim 15-20$ min. We did not design the injection system with a possibility of electronically gradually rewarming the water to a nonanesthetic plane. However, it would be possible to add a small heating unit that would allow the temperature of the water to increase gradually after the injection to let the fish to recover within the same bath.

Additionally, it could be possible to increase the size of the aquarium tank and bed so that more fish could be injected in a single injection process. This type of flexibility in possible system modifications allows the investigator to modify this machine as per their need for anesthesia and recovery change. 
This type of system with gradual cooling and warming could likely be used for performing procedures, such as cerebroventricular microinjections into the adult zebrafish brain that are currently and can only be done using chemical anesthetics. $^{18-21}$

\section{Conclusion}

In this current project, we have designed a novel, low-cost anesthesia and injection system that can be easily manufactured in an investigator's institute or university. It reliably stabilizes and anesthetizes three fish that can be injected under $1 \mathrm{~min}$. This permits a quick reliable method with minimal interaction between the animal and the investigator. Moreover, this would very likely induce the least amount of stress for the fish. Finally, the system also has the potential to be adapted to the different procedural needs of the investigators as they develop new and interesting research questions using the zebrafish model organism.

\section{Acknowledgments}

The authors would like to thank Tulay Arayici for excellent technical assistance with the experiments and Narin Ilgım Ardıç for help with the video production.

This work was supported by a European Molecular Biology Organization (EMBO) Installation Grant to Michelle M. Adams.

\section{Disclosure Statement}

The authors declare no competing financial interests.

\section{References}

1. Stewart A, Cachat J, Suciu C, Hart P, Gaikwad S, Utterback $\mathrm{E}$, et al.: Intraperitoneal Injection As A Method Of Psychotropic Drug Delivery In Adult Zebrafish. In: Zebrafish Neurobehavioral Protocols. Kalueff AV, Cachat JM (eds), pp. 169-179, Humana Press, Totowa, NJ 2011.

2. Pugach EK, Li P, White R, Zon L. Retro-orbital injection in adult zebrafish. J Vis Exp 2009;34:e1645.

3. Kinkel MD, Eames SC, Philipson LH, Prince VE. Intraperitoneal injection into adult zebrafish. J Vis Exp 2010; 42:e2126.

4. Arslan-Ergul A, Halim DO, Adams MM. Bromodeoxyuridine (BrdU) Labeling And Immunohistochemical Detection In Adult Zebrafish Brain Protoc Exch 2013;12:23. Available at: www.nature.com/protocolexchange/protocols/2885 (Accessed Dec 18, 2016).

5. Arslan-Ergul A, Erbaba B, Karoglu ET, Halim DO, Adams MM. Short-term dietary restriction in old zebrafish changes cell senescence mechanisms. Neuroscience 2016;334:64-75.

6. Carter KM, Woodley CM, Brown RS. A review of tricane methanesulfate for anesthesia of fish. Rev Fish Biol Fisheries 2011;21:51-59.

7. Samaee SM, Seyedin S, Varga ZM. An affordable intraperitoneal injection setup for juvenile and adult zebrafish. Zebrafish 2017;14:77-79.
8. Riffat SB, Ma X. Thermoelectrics: a review of present and potential applications. Appl Therm Eng 2003;23:913-935.

9. Wilson JM, Bunte RM, Carty AJ. Evaluation of rapid cooling and tricaine methanesulfonate (MS222) as methods of euthanasia in zebrafish (Danio rerio). JAALAS 2009;48: 785-789.

10. Huang WC, Hsieh YS, Chen IH, Wang $\mathrm{CH}$, Chang HW, Yang CC, et al. Combined use of MS-222 (Tricaine) and isoflurane extends anesthesia time and minimizes cardiac rhythm side effects in adult zebrafish. Zebrafish 2010;7: 297-304.

11. Readman GD, Owen SF, Murrell JC, Knowles TG. Do fish perceive anaesthetics as aversive? PLoS One 2013;8:E73773.

12. Wong D, von Keyserlingk MAG, Richards JG, Weary DM. (2014) Conditioned place avoidance of zebrafish (Danio rerio) to three chemicals used for euthanasia and anaesthesia. PLoS One 2014;9:e88030.

14. Chen K, Wang CQ, Fan YQ, Xie YS, Yin ZF, Xu ZJ, et al. The evaluation of rapid cooling as an anesthetic method for the zebrafish. Zebrafish 2014;11:71-75.

13. Collymore C, Tolwani A, Lieggi C, Rasmussen S. Efficacy and safety of 5 anesthetics in adult zebrafish (Danio rerio). J Am Assoc Lab Anim Sci 2014;53:198-203.

15. Yeh CM, Glöck M, Ryu S. An optimized whole body cortisol quantification method for assesing stress levels in larval zebrafish. PLoS One 2013;8:e79406.

16. Pavlidis M, Sundvik M, Chen YC, Panula P. Adaptive changes in zebrafish brain in dominant-subordinate behavioral context. Behav Brain Res 2011;225:529-537.

17. Barcellos LJG, Ritter F, Kreutz LC, Cericato L. Can zebrafish danio rerio learn about predation risk? the effect of a previous experience on the cortisol response in subsequent encounters with a predator. J Fish Biol 2010;76:1032-1038.

18. Kizil C, Brand M. Cerebroventricular microinjection (CVMII) into adult zebrafish brain is an efficient method for forebrain ventricular cells. PLoS One 2011;6:e27396.

19. Kizil C, Iltzsche A, Brand M. Micromanipulation of gene expression in the adult zebrafish using cerebroventricular microinjection of morpholino oligonucleotides. J Vis Exp 2013;75:e50415.

20. Bhattarai P, Thomas AK, Cosacak MI, Papadimitriou C, Mashkaryan V, Froc C. IL4/STAT6 signaling activates neural stem cell proliferation and neurogenesis upon amyloid- $\beta 42$ aggregation in adult zebrafish brain. Cell Rep 2016;17:941-948.

21. Bhattarai P, Thomas AK, Zhang Y, Kizil C. The effects of aging on amyloid- $\beta 42$-induced neurodgeneration and regeneration in adult zebrafish brain. Neurogenesis 2017;4: e1322666.

Address correspondence to:

Michelle M. Adams, PhD

Interdisciplinary Graduate Program in Neuroscience Aysel Sabuncu Brain Research Center Bilkent University Ankara 06800 Turkey

E-mail: michelle@bilkent.edu.tr 